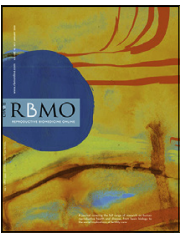

ARTICLE

\title{
How common is real repeated implantation failure? An indirect estimate of the prevalence
}

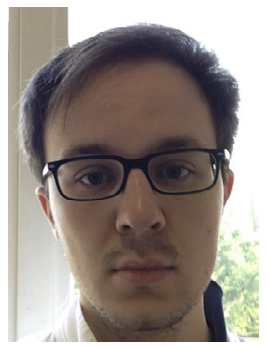

\begin{abstract}
BIOGRAPHY
Andrea Busnelli graduated in Obstetrics and Gynaecology in 2018. He is currently a PhD student in Community Health at the University of Milan, Italy. His activities and research interests cover the whole field of reproductive medicine.
\end{abstract}

Andrea Busnelli, $i^{1, *}$, Marco Reschini ${ }^{2}$, Lucia Cardellicchio ${ }^{1,2}$, Walter Vegetti ${ }^{2}$, Edgardo Somigliana ${ }^{1,2}$, Paolo Vercellini ${ }^{1,2}$

\section{KEY MESSAGE}

The commonly used definition of repeated implantation failure based on three failed attempts in goodprognosis couples seems justified. Healthcare providers and researchers, however, should be aware that couples may be inappropriately labelled with this condition in one out of four cases.

\section{ABSTRACT}

Research question: What is the real prevalence of repeated implantation failure (RIF) and what reliable estimates can be given on the risk of false-positive diagnosis after two or three failed IVF attempts.

Design: A recent theoretical model suggested that commonly used definitions (two or three failed IVF attempts in good-prognosis couples) may expose couples to substantial odds of overdiagnosis and overtreatment. This model, however, was theoretical and based on unproven assumptions that the pregnancy rate in the non-RIF population was $30 \%$ and the prevalence of RIF was $10 \%$. In the present study, we applied this model to real data to distinguish the real prevalence of RIF and to provide more reliable estimates on the risk of false-positive diagnosis after two or three failed IVF attempts. To this aim, we retrospectively selected 1221 good-prognosis couples and evaluated pregnancy rates up to the third cycle.

Results: The clinical pregnancy rate at first, second and third IVF cycle was $52 \%, 41 \%$ and $28 \%$, respectively. A pregnancy rate of $61 \%$ was extrapolated in the non-RIF population and $15 \%$ among women who had experienced RIF. Therefore, the rate of false-positive diagnoses of RIF after two, three and six failed cycles would be $46 \%, 25 \%$, and $2 \%$, respectively.

Conclusions: Our analyses show that estimated prevalence of RIF is $15 \%$. The frequently used definition of RIF based on three failed attempts (but not two) in good-prognosis couples seems justified. Physicians, however, should bear in mind that couples may be inappropriately labelled with this condition in one out of four cases.

\footnotetext{
${ }^{1}$ Department of Clinical Sciences and Community Health, Università degli Studi di Milano, Via Festa del Perdono 7, 20122, Milan, Italy

2 Obstetric amd Gynaecology Department, Fondazione IRCCS Ca' Granda Ospedale Maggiore Policlinico, Via Della Commenda 12, 20122, Milan, Italy
}

\section{KEYWORDS}

infertility

IVF

Repeated implantation failure

RIF

2019 Reproductive Healthcare Ltd. Published by Elsevier Ltd. All rights reserved.

${ }^{*}$ Corresponding author. Infertility Unit Fondazione IRCCS Ca' Granda Ospedale Maggiore Policlinico Via M. Fanti, 6 ,

Milan, 20122, Italy. E-mail address: andrea.busnelli@unimi.it (A Busnelli). https://doi.org/10.1016/j.rbmo.2019.10.014 1472-

6483/@ 2019 Reproductive Healthcare Ltd. Published by Elsevier Ltd. All rights reserved.

Declaration: The study was partly supported by an unrestricted grant from Ferring. 


\section{INTRODUCTION}

$\mathrm{R}$

epeated implantation failure

(RIF) is a debated and

nebulous clinical entity (Evers,

2016; Macklon, 2017; Bashiri

et al., 2018; Bellver and Simón, 2018;

Coughlan, 2018). A plethora of definitions have been provided, variously combining the number of failed attempts (in general two to three) and information on the characteristics of patient cycles and embryos (mainly restricting the definition to couples with good prognosis) (Simon and Laufer, 2012; Coughlan et al., 2014; Polanski et al., 2014). From a clinical perspective, these definitions seem sensible because couples failing to become pregnant after three attempts merit and need re-evaluation and special care. They should be re-counselled on their situation and prognosis, and may benefit from enhanced attention. In these situations, however, over-diagnosis is also probable (Evers, 2016; Somigliana et al., 2018). Given the relatively low rate of success of IVF, a substantial proportion of couples entering an IVF programme are expected to fail three attempts just because of misfortune. Couples who experience repeated failure, however, are extremely vulnerable and may consent to undergo expensive and unsupported procedures that can expose them to undue risks. For this reason, it would be of great relevance to distinguish couples who are just unlucky from those who face real obstacles to success.

Recently, we published a theoretical model based on three basic assumptions: IVF success rate of $30 \%$; RIF considered a dichotomic condition; and prevalence of RIF set at $10 \%$. We showed that, in this situation, the rate of false-positive diagnoses of RIF after two and three failed attempts would be $81 \%$ and $75 \%$, respectively. Even after six cycles, this odd would remain high (51\%). The reliability of the diagnosis of the condition could be partly improved in couples with optimal prognosis (50\% rate of success) or if the postulated prevalence of RIF would be higher than 10\%, but, still, a high number of failed cycles (five to six) would be required for a reliable diagnosis of the condition (Somigliana et al., 2018). Even if plausible, however, this model is theoretical, and the reported estimates must be interpreted with caution.

In this scenario, a significant step forward would be to determine the real prevalence of RIF, i.e. not the proportion of couples who do not achieve pregnancy after two to three cycles but, in contrast, the proportion of those with a real obstacle to conception. In the present study, we aimed to estimate this prevalence by applying the abovementioned model to real data. In other words, we started from observed chances of pregnancy in consecutive cycles to indirectly disentangle the prevalence of RIF and to determine the reliability of this diagnosis according to the number of failed cycles.

\section{MATERIALS AND METHODS}

Clinical charts of women who underwent their first IVF and intracytoplasmic sperm injection (ICSI) cycle between January 2014 and December 2017 at the Infertility Unit of the Fondazione Ca' Granda Ospedale Maggiore Policlinico were reviewed. Patients were included if they fulfilled the following criteria: aged between 18 and 39 years at the start of the first IVF/ICSI cycle; had not undergone any previous IVF cycles; patients had undergone IVF cycles to treat infertility (IVF cycles for genetic indications were excluded); availability of ejaculated sperm (couples with testicular spermatozoa obtained with surgical procedures were excluded); normal uterine cavity (women with unoperated uterine septa, endometrial polyps or submucosal fibroids were excluded); and absence of hydrosalpinx at basal ultrasound. Women who did not obtain viable embryos at any of the included cycles were also excluded. Women could be included up to the third cycle. Overall, these selection criteria aimed to identify a population of patients with a good prognosis and, at the same time, to reduce the drop-out rate (or at least prevent drop-out owing to poor prognosis). Poor prognosis was indeed reported to be associated with a higher rate of dropouts (Gameiro et al., 2012). The study was approved by the local Institutional Review Board on 9 May 2017 (reference number 708). Informed consent was not required as the study was retrospective. All couples referred to our unit, however, were asked to provide consent for the use of their data for research purposes and those declining to provide consent were excluded.

Women were treated as reported in detail elsewhere (Cardellicchio et al., 2017; Benaglia et al., 2018). A cycle was defined as ovarian stimulation leading to oocyte retrieval regardless of the number of subsequent transfers with fresh or frozen embryos that could be carried out. The protocol of embryo vitrification and warming was carried out in accordance with the procedure described by Kuwayama (2007). During the study period, the proportion of embryos surviving warming was equal to 97\% (95\% Cl 96 to $98 \%$ ).

Cycles were supported by the public health system. According to the policy of our unit, women were systematically offered at least three completed IVF cycles. Young women ( $<40$ years) who transferred viable embryos were systematically encouraged to complete this programme. Women could be actively cancelled by physicians before ending the third cycle if no viable embryos were obtained. In the analyses, the cycle was considered successful if a clinical pregnancy (ultrasound visualization of an intrauterine sac even in the absence of fetal heart beating) was documented. Women achieving clinical pregnancies were censored from our analyses regardless of pregnancy outcome. Conversely, biochemical and extrauterine pregnancies were classified as unsuccessful and patients remained in the cohort.

The assumptions of the model used to extrapolate the rate of RIF were as follows: RIF is a dichotomous condition, i.e. couples are either affected or unaffected, affecting $Z$ out of $N$ couples who started the programme; the probability of pregnancy in the nonaffected group ( $Y$ ) was constant over the three cycles; and none of the couples dropped out. On this basis, out of N women entering the IVF programme, the number of pregnancies obtained at first $\left(X_{1}\right)$, second $\left(X_{2}\right)$ and third $\left(X_{3}\right)$ cycles are:

$$
\begin{aligned}
& X_{1}=(N-Z) \star Y \\
& X_{2}=\left(N-X_{1}-Z\right) \star Y \\
& X_{3}=\left(N-X_{1}-X_{2}-Z\right) \star Y
\end{aligned}
$$

Once $X_{1}, X_{2}$ and $X_{3}$ are known, the mathematical problem can be easily solved (three equations with two unknown variables). Even if equation 1 and 2 could be sufficient, we decided to solve the problem using equations 1 and 3 to improve the accuracy of the calculations. $X_{1}, X_{2}$ and $X_{3}$ 


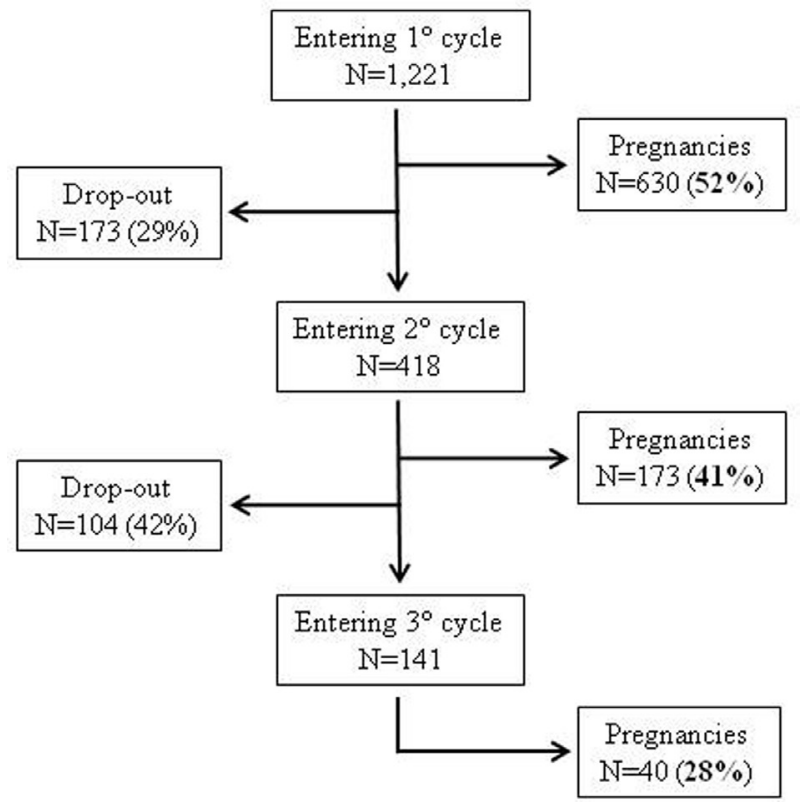

FIGURE 1 Study flow chart. Overall, 843 out of 1221 couples (69\%) obtained a clinical pregnancy $(630+173+40)$ and $277(23 \%)$ dropped out $(173+104)$. could not be directly obtained by real data, however, because the model needs the raw number of pregnancies obtained. Real data are distorted by the presence of dropouts. To overcome this pitfall, we applied the observed pregnancy rates at first, second and third cycle to a virtual cohort of couples. The virtual number of couples entering this simulation $(\mathrm{N})$ was obtained by subtracting from the initial real cohort the total num- ber of couples who subsequently dropped out. This shrewdness consented to extrapolate $X_{1}, X_{2}$ and $X_{3}$ in a theoretical situation of absence of dropouts and, consequently, to infer $Y$ and $Z$.

The Statistical Package for Social Sciences (SPSS 23.0, IBM Corp., USA) was used for all statistical analyses. $P<0.05$ was

\section{TABLE 1 BASELINE CHARACTERISTICS OF THE STUDIED COHORT OF 1221} COUPLES

\begin{tabular}{ll}
\hline Characteristics & Mean $\pm \mathbf{S D}, \mathbf{n}(\%)$ or median (IQR) \\
\hline Age, years & $34.3 \pm 3.6$ \\
\hline BMI, kg/m ${ }^{2}$ & $22.5 \pm 4.2$ \\
\hline Previous deliveries & $91(7 \%)$ \\
\hline Previous pregnancy terminations & $69(6 \%)$ \\
\hline Previous miscarriages & $153(13 \%)$ \\
\hline FSH, IU/I & $6.8[5.5-8.4]$ \\
\hline AMH, ng/ml & $2.2[1.1-3.9]$ \\
\hline AFC & $12[8-17]$ \\
\hline Duration of infertility (months) & $36[24-50]$ \\
\hline Indication to IVF/ICSI & \\
\hline Male factor & $411(34 \%)$ \\
\hline Endometriosis & $200(16 \%)$ \\
\hline Tubal factor & $154(13 \%)$ \\
\hline Unexplained & $400(33 \%)$ \\
\hline Mixed & $56(5 \%)$ \\
\hline
\end{tabular}

Data are reported as mean $\pm S D, n(\%)$ or median (IQR)

a Includes 37 women with endometriosis and 19 women with tubal factor.AFC, antral follicle count; AMH, anti-Müllerian hormone; BMI, body mass index; ICSI, intracytoplasmic sperm injection; SD, standard deviation; $\mathrm{IQR}$, interquartile range. considered statistically significant. Chisquared test, Fisher's exact test, unpaired Student t-test and Mann-Whitney test were used for comparisons, as appropriate. A binomial distribution model was used to estimate the $95 \%$ confidence interval of proportions. On the basis of the expected pregnancy rate and rate of drop-out in our programme, we estimated that recruiting at least 1000 women at the beginning of the programme could allow us to obtain an accuracy of $5 \%$, i.e. a $95 \% \mathrm{Cl}$ of the estimated prevalence of RIF less than $5 \%$. A 4-year period of recruitment (2014-2017) was deemed sufficient to achieve this goal. Exploratory subgroup analyses were run according to age, ovarian reserve and indication. The former two were conducted by dividing the cohort into two groups based on the median. For the indication, four groups were considered, i.e. male infertility, unexplained infertility, endometriosis and tubal factor.

\section{RESULTS}

The initial cohort included 1221 couples. The flow-chart of their IVF course is presented in FIGURE 1. Overall, 277 couples dropped out and did not complete their scheduled programme. The clinical characteristics of the whole cohort is presented in TABLE 1. The outcomes of IVF in the three studied cycles are presented in TABLE 2. The clinical pregnancy rate at first, second and third cycle was $52 \%$ (95\% Cl 49 to 54\%), $41 \%$ (95\% Cl 37 to $46 \%$ ) and 28\% (95\% Cl 21 to $37 \%)$, respectively. Comparison of the characteristics of women who did and did not drop out is presented in Supplementary Table 1. Overall, women who dropped out were more likely to have had a previous child and responded more to ovarian stimulation. We also compared women who achieved a clinical pregnancy after the first cycle with those who did not achieve a clinical pregnancy after the third cycle (Supplementary Table 2). Classical prognostic factors and cycle outcomes significantly differ between these two groups.

Applying our hypothetical model (Somigliana et al., 2018) to the pregnancy rates observed at first, second and third cycle $(52 \%, 41 \%$ and $28 \%$, respectively) it can be inferred that the pregnancy rate in the non-RIF population would be $61 \%$ and the rate of RIF would be $15 \%$ (95\% Cl 13 to $17 \%$ ). Sensitivity analyses based on age, ovarian reserve and indication are presented in TABLE 3. 


\section{TABLE 2 CHARACTERISTICS OF THE IVF/ICSI CYCLES}

\begin{tabular}{|c|c|c|c|}
\hline Characteristics & First cycle & Second cycle & Third cycle \\
\hline Number of women & 1221 & 418 & 141 \\
\hline \multicolumn{4}{|l|}{ Protocol of ovarian stimulation, $n(\%)$} \\
\hline GnRH antagonists & $918(75)$ & $296(71)$ & $96(68)$ \\
\hline Long protocol & $192(16)$ & $69(17)$ & $17(12)$ \\
\hline Flare-up & $111(9)$ & $53(13)$ & $28(20)$ \\
\hline Duration of stimulation, days & $9.3 \pm 2.4$ & $9.3 \pm 2.2$ & $8.9 \pm 1.7$ \\
\hline Total dose of gonadotrophins, IU & $1900[1488-2500]$ & 2100 [1550-2700] & $2400[1800-3000]$ \\
\hline Oocytes retrieved, $n$ & $7[5-11]$ & $6[4-10]$ & $6[4-10]$ \\
\hline Suitable oocytes retrieved, $n$ & $6[4-9]$ & 5 [3-8] & 5 [3-8] \\
\hline Suitable embryos, $n$ & $4[2-6]$ & $3[2-5]$ & $2[1-5]$ \\
\hline Rate of top-quality embryo, $\%$ & $42[41-43]$ & 38 [36-41] & 32 [28-36] \\
\hline \multicolumn{4}{|l|}{ Fertilization technique, $n(\%)$} \\
\hline IVF & $533(44)$ & 165 (39) & $52(37)$ \\
\hline ICSI & $688(56)$ & $253(61)$ & $89(63)$ \\
\hline Women freezing oocytes, n (\%) & $114(9)$ & $25(6)$ & $2(1)$ \\
\hline $\begin{array}{l}\text { Women freezing embryos or blas- } \\
\text { tocysts, } n(\%)\end{array}$ & $641(52)$ & $123(29)$ & $30(21)$ \\
\hline \multicolumn{4}{|l|}{ Time of fresh embryo transfer, $n(\%)$} \\
\hline Not performed & $294(24)$ & $73(17)$ & $16(11)$ \\
\hline $48 \mathrm{~h}$ & $140(11)$ & $67(16)$ & $27(19)$ \\
\hline $72 \mathrm{~h}$ & $707(58)$ & $269(64)$ & $96(68)$ \\
\hline Blastocyst & $80(7)$ & $9(2)$ & $2(1)$ \\
\hline \multicolumn{4}{|l|}{ Cycles with frozen material, $n(\%)$} \\
\hline None & $732(60)$ & $280(67)$ & $111(79)$ \\
\hline 1 & $312(26)$ & $84(20)$ & 19 (13) \\
\hline 2 & $108(9)$ & $35(8)$ & $8(6)$ \\
\hline$\geq 3$ & $69(6)$ & $19(5)$ & $3(2)$ \\
\hline Embryos transferred, $n$ & $1[1-2]$ & $1[1-2]$ & $1[1-2]$ \\
\hline \multicolumn{4}{|l|}{ Embryo transfer, $n(\%)$} \\
\hline 1 & $883(72)$ & $317(76)$ & $126(89)$ \\
\hline 2 & $236(19)$ & $75(18)$ & $11(8)$ \\
\hline$\geq 3$ & $102(8)$ & $26(6)$ & $4(3)$ \\
\hline Clinical pregnancy, n (\%) & $630(52)$ & $173(41)$ & $40(28)$ \\
\hline Live birth, $n(\%)$ & $516(42)$ & $125(30)$ & $30(21)$ \\
\hline \multicolumn{4}{|l|}{ Number of live births, $n(\%)$} \\
\hline Singletons & $494(96)$ & $119(95)$ & $28(93)$ \\
\hline Twins & $22(4)$ & $6(5)$ & $2(7)$ \\
\hline
\end{tabular}

Data are reported as mean $\pm S D, n(\%)$ or median [IQR]

$\mathrm{GnRH}$, gonadotrophin releasing hormones; ICSI, intracytoplasmic sperm injection.

Overall, no major differences emerged with the exception of younger women who had a lower prevalence of RIF. The observed and inferred clinical pregnancy rates at first, second and third cycle are presented in FIGURE 2. The coefficient of correlation Rho between observed and calculated pregnancy rates was 1.0 $(P=0.017)$. In addition, FIGURE 2 shows the predicted pregnancy rates up to the sixth cycle by applying our model.

To ascertain the relationship between the number of failed cycles and the reliability of the diagnosis of RIF, the pregnancy rate in the non-RIF population and the rate of RIF obtained from our analyses (61\% and $15 \%$, respectively) were added to the model. The reliability of the diagnosis of RIF according to the number of failed cycles is presented in FIGURE 3. Specifically, the rate of false- positive diagnoses of RIF after one, two, three, four, five and six cycles would be $69 \%, 46 \%, 25 \%, 12 \%, 5 \%$ and $2 \%$, respectively.

\section{DISCUSSION}

On the basis of our analyses, the estimated prevalence of RIF is $15 \%$, which is higher than previously hypothesized (Somigliana et al., 2018). To the best of our knowledge, this study represents the first attempt to estimate the real prevalence of this condition. Moreover, our data suggest that the magnitude of the odds of RIF is lower in younger women, whereas it is not markedly influenced by ovarian responsiveness and indication to IVF. These analyses on the prevalence of RIF according to the characteristics of the couples are, however, exploratory and deserve to be further investigated in specific studies with larger sample sizes.

The prevalence of RIF emerging from our analyses is strictly related to a model postulated a priori and, as such, is arbitrary. A more rational approach would be to extend the analyses to a higher number of cycles to reach the plateau in the cumulative curve. This is practically unfeasible, however. Previous studies reporting data from national registers in up to six to 10 cycles did not reach a plateau (Malizia et al., 2009; Smith et al., 2015; McLernon et al., 2016; Chambers et al., 2017), and thus a proper analysis would require further extension of the number of cycles. Moreover, and most importantly, only a minority of women underwent more than six cycles in these studies, and this may reflect confounders. Indeed, only couples with a very good prognosis are generally offered more than three to six cycles. Extending the analyses to 10 cycles or more to reach the plateau may actually underestimate the rate of RIF because couples persisting in the programme may be positively selected. For this reason, in our study, we decided to include only good-prognosis patients and to limit the analyses to only three completed cycles. In these circumstances, dropouts could be expected to occur for reasons not related to prognosis. It has to be acknowledged that, in our study, couples who did and did not drop out differed in some characteristics and that, surprisingly, couples who abandoned had 
TABLE 3 SENSITIVITY ANALYSES ACCORDING TO AGE, INDICATION TO IVF AND OVARIAN RESPONSE

\begin{tabular}{|c|c|c|c|c|c|c|c|}
\hline Characteristics & Total number & $\begin{array}{l}\text { Number of } \\
\text { dropouts }\end{array}$ & $\begin{array}{l}\text { Pregnancies at } \\
\text { first cycle (\%) }\end{array}$ & $\begin{array}{l}\text { Pregnancies at } \\
\text { second cycle (\%) }\end{array}$ & $\begin{array}{l}\text { Pregnancies at } \\
\text { third cycle (\%) }\end{array}$ & $\begin{array}{l}\text { Estimated } \\
\text { pure PR (\%) }\end{array}$ & $\begin{array}{l}\text { Estimated RIF } \\
(\% \text { and } 95 \% \mathrm{CI} \text { ) }\end{array}$ \\
\hline \multicolumn{8}{|l|}{ Age } \\
\hline$<35$ years & 572 & 129 & 312 (55) & $81(46)$ & $21(42)$ & 60 & $9(6$ to 12$)$ \\
\hline$\geq 35$ years & 649 & 148 & $318(49)$ & $92(38)$ & $19(21)$ & 61 & 20 (16 to 24$)$ \\
\hline \multicolumn{8}{|l|}{ Indication to IVFa } \\
\hline Male factor & 467 & 109 & $226(48)$ & $73(41)$ & $15(25)$ & 57 & 16 (12 to 20$)$ \\
\hline Endometriosis & 237 & 57 & $115(49)$ & 34 (39) & $13(42)$ & 55 & 11 (6 to 16$)$ \\
\hline Tubal factor & 173 & 38 & $93(54)$ & $27(46)$ & $3(20)$ & 64 & 15 (9 to 21 ) \\
\hline Unexplained & 400 & 83 & $229(57)$ & $46(40)$ & $12(29)$ & 68 & 16 (12 to 20$)$ \\
\hline \multicolumn{8}{|l|}{ Ovarian response } \\
\hline $\begin{array}{l}\text { Less than seven } \\
\text { oocytes }\end{array}$ & 519 & 127 & $222(43)$ & $87(39)$ & $22(27)$ & 51 & 16 (12 to 20$)$ \\
\hline $\begin{array}{l}\text { Seven oocytes or } \\
\text { more }\end{array}$ & 702 & 150 & $408(58)$ & $86(44)$ & $18(31)$ & 68 & 15 (12 to 18$)$ \\
\hline All couples & 1221 & 277 & $630(52)$ & $173(41)$ & $40(28)$ & 61 & 15 (13 to 17$)$ \\
\hline
\end{tabular}

Data are reported as $n(\%)$ or percentage $(95 \% \mathrm{Cl})$.

a Couples with mixed indications were included in more than one category.Pure PR, pregnancy rate in the non-RIF population; RIF, prevalence of repeated implantation failure.

a better prognosis (they were more likely to have had a child and ovarian response was higher). On the other hand, albeit statistically significant, the magnitude of these differences was modest and the effect of these differences on our estimates is presumably limited. Subgroup analyses according to ovarian response did not show any influence of this variable (TABLE 3). Considering potential biases, and, despite our efforts aimed at selecting good-prognosis couples, some significant differences emerged when comparing baseline characteristics of patients who become pregnant in their first cycle and those failing to do so after three cycles. In fact, the frequency of characteristics associated with poor prognosis (older age and lower ovarian response) was higher in women failing to conceive after three cycles.

In our opinion, the most relevant concern with our model is the dichotomic vision of RIF, i.e. the idea that couples can be either affected or unaffected. This view corresponds to a diffuse opinion but seems simplistic. Repeated implantation failure may not be a black and white condition but could be a spectrum ranging from a reduced chance of implantation to a definitive and insurmountable obstacle to implantation. Furthermore, it could also be considered as a time-related condition with differences in endometrial receptivity in different cycles (Bashiri et al., 2018).

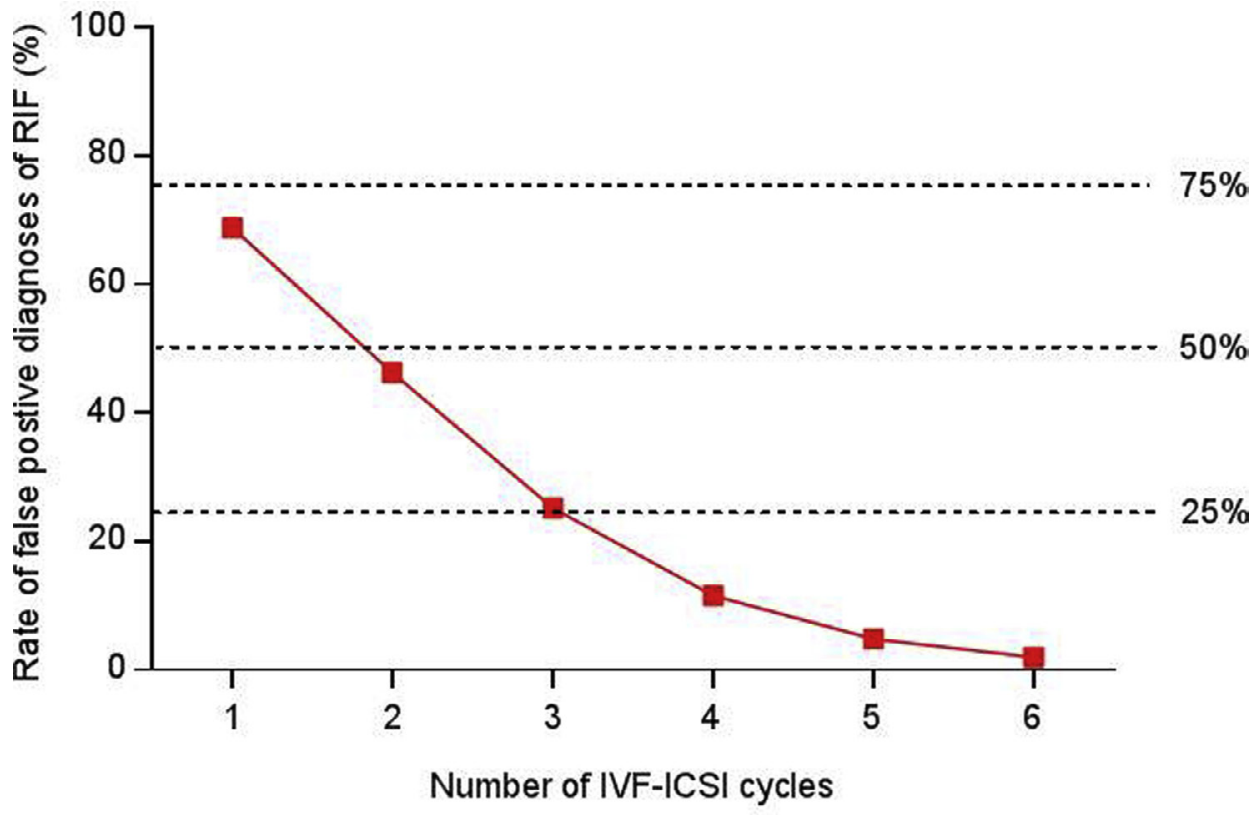

FIGURE 2 Observed and inferred clinical pregnancy rate according to IVF cycle number. Inferred rates were calculated by entering a pregnancy rate in the non-repeated implantation failure (RIF) population of $61 \%$ and a prevalence of RIF of $15 \%$. Observed rates were available only for the first three cycles. Inferences were made up to the sixth cycle. 


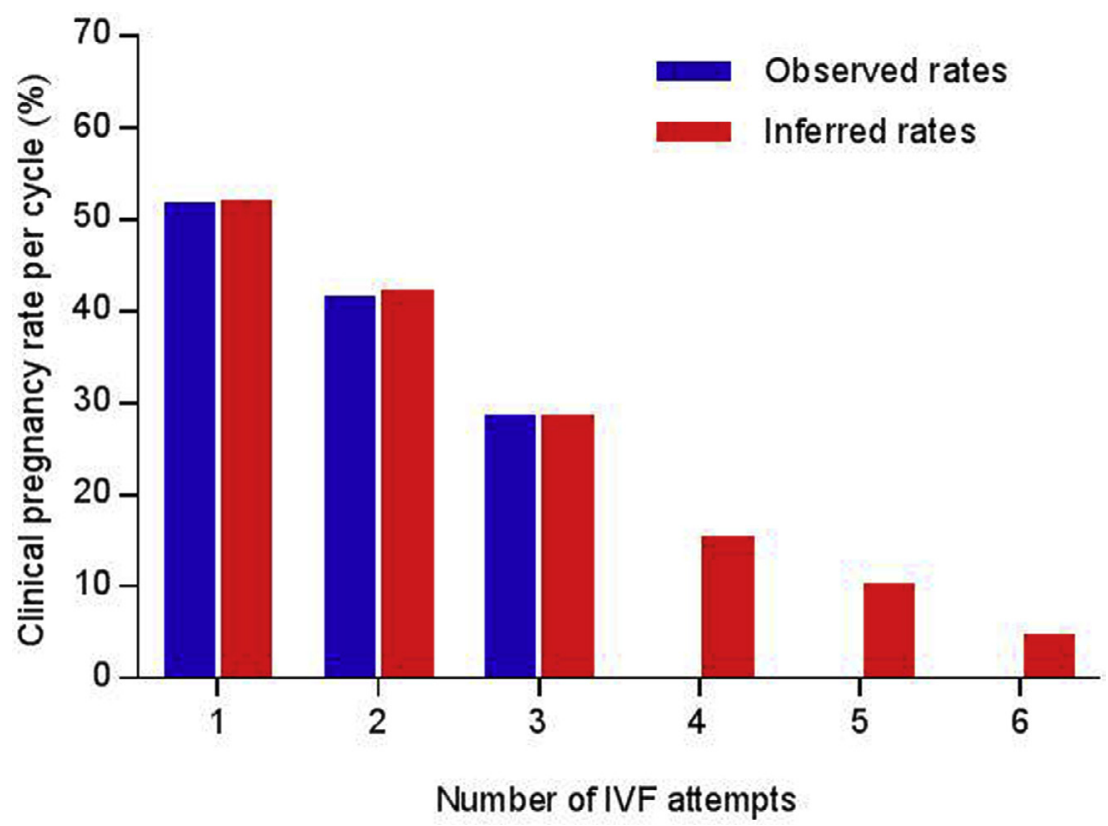

FIGURE 3 Rate of false positive diagnoses of repeated implantation failure (RIF) according to the number of failed cycles. These calculations refer to a situation characterized by a pregnancy rate in the non-RIF population of $61 \%$ and a prevalence of RIF of $15 \%$. After two, three and six failed cycles, the predicted rate of false positive diagnoses is $46 \%, 25 \%$, and $2 \%$, respectively. ICSI, intracytoplasmic sperm injection.

In general, the debate on RIF has always been mislead by the confusion between prognostic factors and real RIF. Shedding light on this issue is crucial and may have important clinical implications. For instance, do older women have a lower chance of pregnancy or are they at higher risk of RIF? In the former situation, a similar cumulative chance of pregnancy can be obtained with a higher number of cycles, whereas, in the latter scenario, extending the number of cycles cannot be expected to overcome the detrimental effects of age. Interestingly, our subgroup analysis on age supports the second interpretation. As previously mentioned, however, results from these secondary analyses were exploratory and the topic deserves to be investigated in other studies.

If one accepts the idea that RIF could be a dichotomic condition, our findings could be used to draw a shared definition of RIF. On the basis of our previous theoretical model (Somigliana et al., 2018) with a clinical pregnancy rate of $61 \%$ and a rate of RIF of $15 \%$, we showed that the rate of false positive diagnoses of RIF after two, three, four, five and six cycles would be $46 \%, 25 \%, 12 \%, 5 \%$ and $2 \%$, respectively (FIGURE 3). This somehow supports the previous threshold of three attempts in good-prognosis women to define RIF, as suggested by most opinion leaders (only one out of four couples would be misclassified) (Coughlan et al., 2014). This definition would be even more valid if one favoured a less trenchant vision of RIF, i.e. not a black and white condition.

Some additional limitations of our model deserve brief mention. First, the dropout rates were elevated, above those generally recorded in assisted reproductive technology units (Gameiro et al., 2013). This is surprising considering that cycles were entirely supported by the public health system. We do not have clear explanations for this. Considering our study design, this high rate is a limitation because it may harbour unknown selection biases. The observation that women who dropped out differed only in a few characteristics is reassuring but does not fully protect our findings from possible confounders. Second, we decided to focus on good-prognosis couples using peculiar selection criteria. Even if most definitions of RIF tend to include patients with an estimated good probability to conceive at baseline, the specific criteria used vary widely (Simon and Laufer, 2012; Coughlan et al., 2014; Polanski et al., 2014). In our study, we decided to select young women (<40 years) who had viable embryos to transfer, and we excluded those with characteristics that are known to be detrimental to implantation (intrauterine pathologies, hydrosalpinx, severe male factor). This choice was, however, based on specific clinical considerations of our group. For the future, drawing a shared and clear definition of these characteristics would be of utmost importance for both clinical practice and research. Third, biochemical and extrauterine pregnancies were considered unsuccessful cycles, whereas early miscarriages were classified as successful events. This decision reflects a peculiar but somehow debatable vision of RIF (incapacity of the embryos to implant in the endometrium) but we have to recognize that this is arbitrary. One may also classify miscarriages as a failure or, conversely, biochemical and extrauterine pregnancies as successes.

In conclusion, our study suggests that RIF may affect one out of eight goodprognosis couples scheduled for IVF. Our findings also support the commonly used definition of RIF based on three failed attempts in good-prognosis couples. Healthcare providers and researchers, however, should be aware that couples may be inappropriately labelled with this condition in one out of four cases. Future studies are required to disentangle whether the prevalence of RIF differs according to the characteristics of the couples.

\section{SUPPLEMENTARY MATERIALS}

Supplementary material associated with this article can be found, in the online version, at doi:10.1016/j. rbmo. 2019.10.014. 


\section{REFERENCES}

Bashiri, A., Halper, K.I., Orvieto, R. Recurrent Implantation Failure-update overview on etiology, diagnosis, treatment and future directions. Reprod. Biol. Endocrinol. 2018; 16: 121

Bellver, J., Simón, C. Implantation failure of endometrial origin: what is new? Curr. Opin. Obstet. Gynecol. 2018; 30: 229-236

Benaglia, L., Busnelli, A., Biancardi, R., Vegetti, W., Reschini, M., Vercellini, P., Somigliana, E. Oocyte retrieval difficulties in women with ovarian endometriomas. Reprod. Biomed. Online 2018; 37: 77-84

Cardellicchio, L., Reschini, M., Paffoni, A., Guarneri, C., Restelli, L., Somigliana, E., Vegetti, W. Frozenthawed blastocyst transfer in natural cycle: feasibility in everyday clinical practice. Arch. Gynecol. Obstet. 2017; 295: 1509-1514

Chambers, G.M., Paul, R.C., Harris, K., Fitzgerald, O., Boothroyd, C.V., Rombauts, L., Chapman, M.G., Jorm, L. Assisted reproductive technology in Australia and New Zealand: cumulative live birth rates as measures of success. Med. J. Aust. 2017; 207: 114-118

Coughlan, C., Ledger, W., Wang, Q., Liu, F., Demirol, A., Gurgan, T., Cutting, R., Ong, K., Sallam, H., Li, T.C. Recurrent implantation failure: definition and management. Reprod. Biomed. Online 2014; 28: 14-38

Coughlan, C. What to do when good-quality embryos repeatedly fail to implant. Best Pract. Res. Clin. Obstet. Gynaecol. 2018; 53: 48-59

Evers, J.L. Is RIF rife? Hum Reprod 2016; 31: 2661

Gameiro, S., Boivin, J., Peronace, L., Verhaak, C.M. Why do patients discontinue fertility treatment? A systematic review of reasons and predictors of discontinuation in fertility treatment. Hum. Reprod. Update 2012; 18: 652-669

Gameiro, S., Verhaak, C.M., Kremer, J.A., Boivin, J. Why we should talk about compliance with assisted reproductive technologies (ART): a systematic review and meta-analysis of ART compliance rates. Hum. Reprod. Update 2013; 19: $124-135$

Kuwayama, M. Highly efficient vitrification for cryopreservation of human oocytes and embryos: the Cryotop method. Theriogenology 2007; 67: 73-80

Macklon, N. Recurrent implantation failure is a pathology with a specific transcriptomic signature. Fertil. Steril. 2017; 108: 9-14

Malizia, B.A., Hacker, M.R., Penzias, A.S

Cumulative live-birth rates after in vitro fertilization. N. Engl. J. Med. 2009; 360 236-243
McLernon, D.J., Maheshwari, A., Lee, A.J. Bhattacharya, S. Cumulative live birth rates after one or more complete cycles of IVF: a population-based study of linked cycle data from 178,898 women. Hum. Reprod. 2016; 31 : 572-581

Polanski, L.T., Baumgarten, M.N., Quenby, S. Brosens, J., Campbell, B.K., Raine-Fenning, N.J. What exactly do we mean by 'recurrent implantation failure'? A systematic review and opinion. Reprod. Biomed. Online 2014; 28: 409-423

Simon, A., Laufer, N. Repeated implantation failure: clinical approach. Fertil. Steril. 2012; 97: 1039-1043

Smith, A.D.A.C., Tilling, K., Nelson, S.M., Lawlor, D.A. Live-Birth Rate Associated With Repeat In Vitro Fertilization Treatment Cycles. JAMA 2015: 314: 2654-2662

Somigliana, E., Vigano, P., Busnelli, A., Paffoni, A. Vegetti, W., Vercellini, P. Repeated implantation failure at the crossroad between statistics, clinics and over-diagnosis. Reprod. Biomed. Online 2018; 36: 32-38

Received 30 May 2019; received in revised form 12 September 2019; accepted 21 October 2019. 